\title{
Symmetrization and minimax principles
}

\author{
Jean Van Schaftingen
}

July 20, 2004

\begin{abstract}
We develop a method to prove that some critical levels for functionals invariant by symmetry obtained by minimax methods without any symmetry constraint are attained by symmetric critical points. It is used to investigate the symmetry properties of solutions of elliptic partial differential equations with Dirichlet or Neumann boundary conditions. It is also an alternative to concentration-compactness for some symmetric elliptic problems.
\end{abstract}

\section{Introduction}

We are concerned by symmetry properties of symmetric elliptic partial differential equations. Our model problem is

$$
\begin{cases}-\Delta u=f(|x|, u) & \text { in } \Omega, \\ u=0 & \text { on } \partial \Omega,\end{cases}
$$

where $\Omega$ is a ball and $u$ is a real-valued function. When the function $f$ is decreasing in $|x|$ and $u$ is a positive solution continuous up to the boundary, then Gidas, Ni and Nirenberg's celebrated result $[6,7]$ says that $u$ is radial and $\frac{\partial u}{\partial r}<0$.

Solutions of (1.1) can be obtained as critical points of the Euler-Lagrange functional $\varphi$ defined on the Sobolev space $H_{0}^{1}(\Omega)$ by

$$
\varphi(u)=\int_{\Omega} \frac{|\nabla u|^{2}}{2}-F(|x|, u) d x
$$

where $F(r, t)=\int_{0}^{t} f(r, s) d s$. In particular one can inquire about the properties of the minimizers of $\varphi$. The Schwarz symmetrization maps a nonnegative function $u \in H_{0}^{1}(\Omega)$ to a more symmetric one $u^{*}$. It can be shown that if $\frac{\partial f}{\partial r} \leqslant 0$, then $\varphi\left(u^{*}\right) \leqslant \varphi(u)$. This proves that if there is a minimizer, then there is a symmetric minimizer. If $\varphi$ is Gateaux-differentiable, then the minimizer is a critical point. Similarly, using the spherical cap symmetrization, one can ensure that without any sign restriction on $u$ or on $\frac{\partial f}{\partial r}$, the minimum of $\varphi$ is attained by a function which depends only on the radius and of one angular variable [11].

Continuous symmetrization - a homotopy linking a function to its symmetrization was used by Brock in order to prove that if $\frac{\partial f}{\partial r} \leqslant 0$, then any nonnegative critical point is locally symmetric, i.e. its domain is the union of annuli on which it is radial and of a set on which $\nabla u=0$ almost everywhere [3].

In this paper we consider critical points obtained by minimax principles. We modify a general minimax principle of Willem in order to obtain Palais-Smale sequences whose elements are 
more and more symmetric. This can be applied to prove that some critical levels are achieved by symmetric functions. It also provides an alternative to concentration-compactness.

The paper is organized as follows. Section 2 is devoted to the definition and properties of symmetrizations and polarizations. We briefly recall the classical properties needed for our purpose. In particular, the Schwarz symmetrization and the spherical cap symmetrization can be both approximated by polarizations. We prove that polarizations are continuous in Sobolev spaces. The essential properties are summarized in an axiomatic framework (section 2.4) for the Schwarz symmetrization and the spherical cap symmetrization. Under these assumptions, a homotopy linking a polarization of a function with its symmetrization is constructed. These axioms are easily verified for many variants, e.g. problems in SobolevOrlicz spaces and in weighted spaces, and approximation of the Schwarz symmetrization by Steiner symmetrizations.

Section 3 is devoted to our symmetric minimax principle (Theorem 3.5) in the abstract framework of section 2.4. The proof is based on a minimax principle of Willem [15]. The idea of the proof is to replace a path by its symmetrization. The main difficulty is the fact that symmetrizations are not continuous in Sobolev spaces; it is overcome by the approximation of the symmetrization by polarizations.

Finally, section 4 gives examples of applications to semi-linear elliptic partial differential equations. We prove the symmetry of critical points at some critical levels obtained by the mountain pass Theorem of Ambrosetti and Rabinowitz and by Rabinowitz's linking Theorem. We also show how the symmetric minimax principle provides an alternative to concentrationcompactness methods in symmetric settings.

All the results in this paper hold for partial symmetrizations $((N, k)$-Steiner symmetrization or $k$-spherical cap symmetrizations). For the sake of clarity, the exposition is made for the Schwarz and $(N-1)$-spherical cap symmetrization, but this restriction can always be removed with no modification in the arguments. Similarly, the results of section 4.1 concerning the spherical cap symmetrization remain valid without any modification for the Neumann boundary conditions.

\section{Symmetrization and polarization}

\subsection{Schwarz symmetrization}

For $f: A \rightarrow \overline{\mathbb{R}}=\mathbb{R} \cup\{-\infty,+\infty\}$ and $c \in \overline{\mathbb{R}}$, let $\{u>c\}=\{x \in A \mid u(x)>c\}$. The set of infinitely differentiable (resp. continuous) functions whose support is compact in $\Omega \subseteq \mathbb{R}^{N}$ is denoted $\mathcal{D}(\Omega)(\operatorname{resp} . \mathcal{K}(\Omega))$.

Definition 2.1. The Schwarz symmetrization of a set $A \subset \mathbb{R}^{N}$ is the unique open ball centered at the origin $A^{*}$ such that $\mathcal{L}^{N}\left(A^{*}\right)=\mathcal{L}^{N}(A)$, where $\mathcal{L}^{N}$ denotes the $N$-dimensional outer Lebesgue measure. If $\mathcal{L}^{N}(A)=0$, then $A^{*}=\phi$ while $A^{*}=\mathbb{R}^{N}$ if $\mathcal{L}^{N}(A)=\infty$.

Definition 2.2. The Schwarz symmetrization of a measurable nonnegative function $u: \Omega \rightarrow$ $\overline{\mathbb{R}}$ is the unique function $u^{*}: \Omega^{*} \rightarrow \overline{\mathbb{R}}$ such that for all $c \in \mathbb{R}$,

$$
\left\{u^{*}>c\right\}=\{u>c\}^{*}
$$

Remark 2.3. The function $u^{*}$ is also characterized by $u^{*}(y)=\sup \left\{c \in \mathbb{R} \mid y \in\{u>c\}^{*}\right\}$. 
Definition 2.4. A measurable function $u$ vanishes at the infinity if for all $\varepsilon>0, \mathcal{L}^{N}(\{|u|>\varepsilon\})<$ $\infty$.

Definition 2.5. A function is admissible for the Schwarz symmetrization if it is nonnegative and it vanishes at the infinity.

Proposition 2.6. If $u: \Omega \rightarrow \overline{\mathbb{R}}$ is admissible, then $u^{*}$ is admissible and for any Borel measurable function $f: \mathbb{R}^{+} \rightarrow \mathbb{R}^{+}$such that $f(0)=0$,

$$
\int_{\mathbb{R}^{N}} f\left(u^{*}(x)\right) d x=\int_{\mathbb{R}^{N}} f(u(x)) d x .
$$

In particular, if $u \in L^{p}(\Omega)$ is nonnegative, then $u^{*} \in L^{p}\left(\Omega^{*}\right)$ and $\|u\|_{p}=\left\|u^{*}\right\|_{p}$.

Remark 2.7. The Steiner symmetrization is an analogue of the Schwarz symmetrization that symmetrizes functions only with respect to certain variables. The $(k, N)$-Steiner symmetrization of a set $A \in \mathbb{R}^{N}$ is the unique set $A^{*}$ such that for all $x^{\prime \prime} \in \mathbb{R}^{N-k},\left\{x^{\prime} \in \mathbb{R}^{k} \mid\left(x^{\prime}, x^{\prime \prime}\right) \in\right.$ $\left.A^{*}\right\}=\left\{x^{\prime} \in \mathbb{R}^{k} \mid\left(x^{\prime}, x^{\prime \prime}\right) \in A\right\}^{*}$. (The $*$ on the right-hand side denotes the Schwarz symmetrization in $\mathbb{R}^{k}$ of Definition 2.1.) It is extended to functions as in Definition 2.2; Proposition 2.6 still holds. Steiner-symmetrized functions have cylindrical symmetry: they can be written as $u^{*}\left(x^{\prime}, x^{\prime \prime}\right)=v\left(\left|x^{\prime}\right|, x^{\prime \prime}\right)$ where $v\left(\cdot, x^{\prime \prime}\right)$ is a decreasing function for each $x^{\prime \prime} \in \mathbb{R}^{N-k}$.

\subsection{Spherical cap symmetrization}

The spherical cap symmetrization is defined following Sarvas [9] (see also $[11,13,14]$ ).

Definition 2.8. Let $P \in \partial B(0,1) \cap \mathbb{R}^{N}$. The spherical cap symmetrization of the set $A$ with respect to $P$ is the unique set $A^{*}$ such that $A^{*} \cap\{0\}=A \cap\{0\}$ and, for any $r>0$,

$$
\begin{aligned}
A^{*} \cap \partial B(0, r) & =B_{g}(r P, \rho) \cap \partial B(0, r) \quad \text { for some } \rho \geqslant 0, \\
\mathcal{H}^{N-1}\left(A^{*} \cap \partial B(0, r)\right) & =\mathcal{H}^{N-1}(A \cap \partial B(0, r)),
\end{aligned}
$$

where $\mathcal{H}^{N-1}$ is the outer Hausdorff $(N-1)$-dimensional measure and $B_{g}(r P, \rho)$ denotes the geodesic ball on the sphere $\partial B(0, r)$ of center $r P$ and radius $\rho$. (By definition, $B_{g}(r P, 0)=\phi$.)

Definition 2.9. The spherical cap symmetrization of a function $u: \Omega \rightarrow \overline{\mathbb{R}}$ is the unique function $u^{*}: \Omega^{*} \rightarrow \overline{\mathbb{R}}$ such that, for all $c \in \mathbb{R}$,

$$
\left\{u^{*}>c\right\}=\{u>c\}^{*} .
$$

The result of a spherical cap symmetrization is a function that depends on two variables: $u^{*}(x)=v(|x|, P \cdot x)$, where $v(r, \cdot)$ is a nondecreasing function for any $r \geqslant 0$.

Definition 2.10. A set $\Omega \subset \mathbb{R}^{N}$ is invariant with respect to $*$ if $\Omega^{*}=\Omega$. It is totally invariant if $\Omega^{*}=\Omega$ and $\left(\mathbb{R}^{N} \backslash \Omega\right)^{*}=\left(\mathbb{R}^{N} \backslash \Omega\right)$.

Definition 2.11. A function $u: \Omega \rightarrow \overline{\mathbb{R}}$ is admissible for the spherical cap symmetrization if it is measurable and either $\Omega$ is totally invariant or $u$ is nonnegative.

As for the Schwarz symmetrization, we have 
Proposition 2.12. If $u: \Omega \rightarrow \overline{\mathbb{R}}$ is admissible, then $u^{*}$ is measurable and for any Borel measurable function $f: \mathbb{R}^{+} \times \overline{\mathbb{R}} \rightarrow \mathbb{R}^{+}$

$$
\int_{\Omega} f\left(|x|, u^{*}(x)\right) d x=\int_{\Omega^{*}} f(|x|, u(x)) d x .
$$

In particular, if $u \in L^{p}(\Omega)$, then $u^{*} \in L^{p}\left(\Omega^{*}\right)$.

Remark 2.13. The equivalent of Steiner symmetrization for the spherical cap symmetrization is the $k$-spherical cap symmetrization with respect to $P \in \mathbb{R}^{k+1}$. The process is the same as in Remark 2.7 and yields symmetrized functions of the form $u^{*}\left(x^{\prime}, x^{\prime \prime}\right)=v\left(\left|x^{\prime}\right|, P \cdot x^{\prime}, x^{\prime \prime}\right)$.

\subsection{Polarizations}

Definition 2.14. A set $H \subset \mathbb{R}^{N}$ is a polarizer if it is a closed affine half-space of $\mathbb{R}^{N}$, i.e. $H$ is the set of all points verifying $a \cdot x \leqslant b$ for some $a \in \mathbb{R}^{k}, b \in \mathbb{R},|a|_{2}=1$.

Notation 2.15. For any $x \in \mathbb{R}^{N}$ and any polarizer $H \subseteq \Omega, x_{H}$ denotes the reflection of $x$ with respect to $\partial H$. With the notation of Definition 2.14, $x_{H}=x-2(a \cdot x-b) a$.

Definition 2.16. The polarization of a function $u: \mathbb{R}^{N} \rightarrow \mathbb{R}$ by the polarizer $H$ is the function $u^{H}: \Omega \rightarrow \mathbb{R}$, with

$$
u^{H}(x)= \begin{cases}\max \left\{u(x), u\left(x_{H}\right)\right\} & \text { if } x \in H, \\ \min \left\{u(x), u\left(x_{H}\right)\right\} & \text { if } x \notin H .\end{cases}
$$

Definition 2.17 (Extended polarizers). The set of polarizers is compactified by the addition of two polarizers at the infinity, defined by $u^{H_{+\infty}}=u_{+}$and $u^{H_{-}}=-u_{-}$, such that $H_{n} \rightarrow H_{+\infty}$ if $b_{n} \rightarrow \infty$ and $H_{n} \rightarrow H_{-\infty}$ if $b_{n} \rightarrow-\infty$ in the representation of Definition 2.14. The compactified set of polarizers is denoted $\mathcal{H}$ and is homeomorphic to $S^{N}$.

Definition 2.18. If $H \in \mathcal{H}_{*}$ and $\Omega \subset \mathbb{R}^{N}$, the polarization of $u: \Omega \rightarrow \overline{\mathbb{R}}$ with respect to $H$ is defined as $u^{H}=\left.\tilde{u}^{H}\right|_{\Omega}$, where $\tilde{u}$ is the extension of $u$ to $\mathbb{R}^{N}$ by 0 outside of $\Omega$.

Proposition 2.19. Let $H \in \mathcal{H}$. Suppose $\Omega=\Omega^{H} \subseteq \mathbb{R}^{N}, u, v: \Omega \rightarrow \overline{\mathbb{R}}$ are measurable and nonnegative.

If $g: \Omega \times \overline{\mathbb{R}} \rightarrow \overline{\mathbb{R}}^{+}$is a Borel measurable function such that $g\left(x_{H}, s\right)=g(x, s)$ for each $(x, s) \in \Omega \times \overline{\mathbb{R}}$, then

$$
\int_{\Omega} g\left(x, u^{H}\right) d x=\int_{\Omega} g(x, u) d x .
$$

If $G: \Omega \times \overline{\mathbb{R}} \times \overline{\mathbb{R}} \rightarrow \overline{\mathbb{R}}^{+}$is a Borel measurable function such that $G\left(x_{H}, s, t\right)=G(x, s, t)$ for each $(x, s, t) \in \Omega \times \overline{\mathbb{R}}$ and for any $x \in \Omega, a \leqslant b$ and $c \leqslant d, G(x, a, c)+G(x, b, d) \geqslant$ $G(x, a, d)+G(x, b, c)$, then

$$
\int_{\Omega} G\left(x, u^{H}, v^{H}\right) d x \geqslant \int_{\Omega} G(x, u, v) d x .
$$

In particular, $\left\|u^{H}-v^{H}\right\|_{p} \leqslant\|u-v\|_{p}$.

If $u \in W_{0}^{1, p}(\Omega)$, then $u \in W_{0}^{1, p}(\Omega)$ and $\left\|\nabla u^{H}\right\|_{p}=\|\nabla u\|_{p}$.

If moreover, $\left(\mathbb{R}^{N} \backslash \Omega\right)^{H}=\mathbb{R}^{N} \backslash \Omega$, the results remain valid without any sign restriction on $u$ and $v$ and if $u \in W^{1, p}(\Omega)$, then $u^{H} \in W^{1, p}(\Omega)$ and $\left\|\nabla u^{H}\right\|_{p}=\|\nabla u\|_{p}$. 
For any symmetrization $*$ defined above, the subset of admissible functions in a function space $Y$ is denoted $Y_{*}$, and there is a set $\mathcal{H}_{*} \subset \mathcal{H}$ of polarizers such that for any admissible function $u: \Omega \rightarrow \overline{\mathbb{R}}$

$$
u=u^{*} \Longleftrightarrow \forall H \in \mathcal{H}_{*}, u=u^{H} .
$$

If $*$ is the $(N, k)$-Steiner symmetrization,

$$
\mathcal{H}_{*}=\left\{H \in \mathcal{H} \mid\{0\} \times \mathbb{R}^{N-k} \subset H \text { or } H=H_{+\infty}\right\} .
$$

If $*$ is the $k$-spherical cap symmetrization,

$$
\mathcal{H}_{*}=\left\{H \in \mathcal{H} \mid \mathbb{R}^{+} \times\{0\} \times \mathbb{R}^{N-k-1} \subset H \text { and }\{0\} \times \mathbb{R}^{N-k-1} \subset \partial H\right\} .
$$

Because polarizations are contractions in $L^{p}\left(\mathbb{R}^{N}\right)$, for any $H \in \mathcal{H}_{*}$ and $u \in L^{p}\left(\mathbb{R}^{N}\right)$, $\left\|u^{H}-u^{*}\right\|_{p} \leqslant\left\|u-u^{*}\right\|_{p}$. In fact they can approximate symmetrizations: in [13], it was shown:

Theorem 2.20. For any symmetrization $*$, there exists a sequence of polarizers $\left(H_{m}\right)_{m \geqslant 1} \subset$ $\mathcal{H}_{*}$ such that, for any $1 \leqslant p<\infty, \Omega \subset \mathbb{R}^{N}$ invariant with respect to $*$ and $u \in L_{*}^{p}(\Omega)$, the sequence $u_{m}=u^{H_{1} \cdots H_{m}}$ converges to $u^{*}$ :

$$
\lim _{m \rightarrow \infty}\left\|u_{m}-u^{*}\right\|_{p}=0 .
$$

Theorem 2.20 was proved for a fixed function by Brock and Solynin for the Steiner symmetrizations [4] and by Smets and Willem for the spherical cap symmetrization [11].

Lemma 2.21. If $1 \leqslant p<\infty$, the map

$$
\mathfrak{h}: \overline{\mathcal{H}} \times L^{p}\left(\mathbb{R}^{N}\right) \rightarrow L^{p}\left(\mathbb{R}^{N}\right):(H, u) \mapsto u^{H}
$$

is continuous at $(u, H)$ if and only if $(u, H) \in \mathcal{H}\left(L^{p}\left(\mathbb{R}^{N}\right)\right)$, where

$$
\mathcal{H}(X)=\left\{(u, H) \in X \times \overline{\mathcal{H}} \mid u \geqslant 0 \text { if } H=H_{+\infty} \text { and } u \leqslant 0 \text { if } H=H_{-\infty}\right\} .
$$

Proof. If $(u, H)$ and $(v, L)$ are in $\mathcal{H}\left(L^{p}\left(\mathbb{R}^{N}\right)\right)$, then

$$
\left\|u^{H}-v^{L}\right\|_{p} \leqslant\left\|u^{H}-v^{H}\right\|_{p}+\left\|v^{H}-v^{L}\right\|_{p} .
$$

The first term is bounded by $\|u-v\|_{p}$ because polarizations are nonexpansive. For the second term, since polarizations are nonexpansive we can suppose without loss of generality that $v$ is a compactly supported continuous function. In the latter case $v^{H} \rightarrow v^{L}$ uniformly on $\mathbb{R}^{N}$ if $(v, L) \in \mathcal{H}\left(\mathcal{K}\left(\mathbb{R}^{N}\right)\right)$ and thus in $L^{p}\left(\mathbb{R}^{N}\right)$.

Proposition 2.22. If $*$ is a symmetrization, $\Omega$ is open and invariant with respect to $*$, then the map $\mathfrak{h}$ is continuous from $L_{*}^{p}(\Omega) \times \mathcal{H}_{*}$ to $L_{*}^{p}(\Omega)$.

Proof. This is a direct consequence of Lemma 2.21 and of Theorem 2.20.

The continuity in Sobolev spaces relies on the next standard lemma.

Lemma 2.23. Let $1<p<\infty,\left(u_{n}\right)_{n \in \mathbb{N}}$ and $u$ be in $W^{1, p}(\Omega)$ and $|\cdot|$ be a strictly convex norm in $\mathbb{R}^{N}$. Then $u_{n} \rightarrow u$ in $W^{1, p}(\Omega)$ if and only if $u_{n} \rightarrow u$ in $L^{p}(\Omega)$ and $\left\|\left|\nabla u_{n}\right|\right\|_{p} \rightarrow\||\nabla u|\|_{p}$. 
Proof. This is a consequence of the strict convexity of the norm $\||\cdot|\|_{p}$.

Proposition 2.24. If $*$ is a symmetrization, $\Omega$ is open and is invariant with respect to $*$ and $1<p<\infty$, the map $\mathfrak{h}$ is continuous from $W_{0,+}^{1, p}(\Omega) \times \mathcal{H}_{*}$ to $W_{0}^{1, p}(\Omega)$.

Moreover, if $\Omega$ is totally invariant with respect to $*$ and $*$ is a spherical cap symmetrization, the map $\mathfrak{h}$ is continuous from $W_{0}^{1, p}(\Omega) \times \mathcal{H}_{*}$ to $W_{0}^{1, p}(\Omega)$ and from $W^{1, p}(\Omega) \times \mathcal{H}_{*}$ to $W^{1, p}(\Omega)$.

Proof. This is a consequence of Lemma 2.23, together with Proposition 2.22 and the fact that $\left\|\left|\nabla u^{H}\right|\right\|_{p}=\||\nabla u|\|_{p}$

\subsection{Abstract symmetrizations and polarizations}

Assumption 2.25. Let $X, V$ be Banach spaces, $*: S \subset X \rightarrow V: u \mapsto u^{*}, \mathcal{H}_{*}$ be a path-connected topological space and $\mathfrak{h}: S \times \mathcal{H}_{*} \rightarrow S:(u, H) \mapsto u^{H}$. Assume

(i) $X$ is continuously embedded in $V$,

(ii) the mapping $\mathfrak{h}$ is continuous,

(iii) for each $u \in S$ and $H \in \mathcal{H}_{*}, u^{* H}=u^{H *}=u^{*}$ and $u^{H H}=u^{H}$,

(iv) there is a sequence $\left(H_{m}\right)_{m \geqslant 1} \subset \mathcal{H}_{*}$ such that for each $u \in S, u^{H_{1} \ldots H_{m}} \rightarrow u^{*}$ in $V$ as $m \rightarrow \infty$

(v) for each $u, v \in S$ and $H \in \mathcal{H}_{*},\left\|u^{H}-v^{H}\right\|_{V} \leqslant\|u-v\|_{V}$.

Example 2.26 (Schwarz symmetrization for nonnegative functions). Let $\Omega=B(0,1) \subset \mathbb{R}^{N}$, $\left.X=W_{0}^{1, p}(\Omega), V=\left(L^{p} \cap L^{p^{*}}\right)(\Omega)\right)$, with $p^{*}=N p /(N-p), S$ be the set of nonnegative functions of $W_{0}^{1, p}(\Omega)$, * denote the Schwarz symmetrization and $\mathcal{H}_{*}$ be defined as above. Assumption 2.25 is satisfied by Proposition 2.19, Theorem 2.20 and Proposition 2.24.

Example 2.27 (Schwarz symmetrization). Let $\Omega=B(0,1) \subset \mathbb{R}^{N}, X=W_{0}^{1, p}(\Omega), V=\left(L^{p} \cap\right.$ $\left.\left.L^{p^{*}}\right)(\Omega)\right)$, with $p^{*}=N p /(N-p), S=W_{0}^{1, p}(\Omega), u^{*}=|u|^{\star}$ where $\star$ denotes the Schwarz symmetrization and $\mathcal{H}_{*}$ is defined as above for the Schwarz symmetrization, but $\mathfrak{h}(u, H)=$ $|u|^{H}$. Assumption 2.25 is satisfied by Proposition 2.19, Theorem 2.20 and Proposition 2.24.

Example 2.28 (Spherical cap symmetrization with Dirichlet boundary condition). Let $\Omega \subset \mathbb{R}^{N}$ be a ball or an annulus, $\left.X=W_{0}^{1, p}(\Omega), V=\left(L^{p} \cap L^{p^{*}}\right)(\Omega)\right)$, with $p^{*}=N p /(N-p)$, * denote the spherical cap symmetrization and $\mathcal{H}_{*}$ be defined as above. Assumption 2.25 is satisfied by Proposition 2.19, Theorem 2.20 and Proposition 2.24.

Example 2.29 (Spherical cap symmetrization with Neumann boundary condition). Let $\Omega \subset \mathbb{R}^{N}$ be a ball or an annulus, $\left.X=W^{1, p}(\Omega), V=\left(L^{p} \cap L^{p^{*}}\right)(\Omega)\right)$, with $p^{*}=N p /(N-p)$, * denote the spherical cap symmetrization and $\mathcal{H}_{*}$ be defined as above. Assumption 2.25 is satisfied by Proposition 2.19, Theorem 2.20 and Proposition 2.24 .

Example 2.30 (Schwarz symmetrization approximated by Steiner symmetrization). Let $\Omega=$ $\left.B(0,1) \subset \mathbb{R}^{N}, X=W_{0}^{1, p}(\Omega), V=\left(L^{p} \cap L^{p^{*}}\right)(\Omega)\right)$, with $p^{*}=N p /(N-p), S$ be the set of nonnegative function of $W_{0}^{1, p}(\Omega)$, * denote the Schwarz symmetrization, $\mathcal{H}_{*}$ denote the set of hyperplanes passing through the origin and $u^{H}$ be the Steiner symmetrization with respect to $H$. Assumption 2.25 is satisfied (see [5] and [13]).

Proposition 2.31. Under Assumption 2.25, for any $u, v \in S,\left\|u^{*}-v^{*}\right\|_{V} \leqslant\|u-v\|_{V}$. 
Proof. By Assumption 2.25, for any $m \geqslant 1$,

$$
\begin{array}{r}
\left\|u^{*}-v^{*}\right\|_{V} \leqslant\left\|u^{*}-u^{H_{1} \ldots H_{m}}\right\|_{V}+\left\|u^{H_{1} \ldots H_{m}}-v^{H_{1} \ldots H_{m}}\right\|_{V}+\left\|v^{H_{1} \ldots H_{m}}-v^{*}\right\|_{V} \\
\leqslant\left\|u^{*}-u^{H_{1} \ldots H_{m}}\right\|_{V}+\|u-v\|_{V}+\left\|v^{H_{1} \ldots H_{m}}-v^{*}\right\|_{V} .
\end{array}
$$

The conclusion comes from the property (iv) as $m \rightarrow \infty$.

Proposition 2.32. Under Assumption 2.25, for any $H_{0} \in \mathcal{H}_{*}$, there exists a continuous mapping $(u, t) \in S \times \mathbb{R}^{+} \mapsto u^{t}$ such that $\lim _{t \rightarrow \infty} u^{t}=u^{*}$ in $V$. Furthermore, for each $t \geqslant 0$, there exists $H_{t} \in \mathcal{H}_{*}$ such that $u^{t}=u^{H_{0} H_{1} \cdots H_{\lfloor t\rfloor} H_{t}}$, where $\lfloor t\rfloor$ denotes the largest integer less or equal to $t$.

Proof. Let $H_{t}$ be a such that $t \mapsto H_{t}$ is continuous in $\mathcal{H}_{*}$. For $t \in[n-1, n], n \in \mathbb{N}$, let

$$
u^{t}=u^{H_{0} \cdots H_{n-1} H_{t}} .
$$

This map is well-defined since $u^{n}=u^{H_{0} \cdots H_{n-1} H_{n}}=u^{H_{0} \cdots H_{n-1} H_{n} H_{n}}$. It is clear that for any $u \in V$

$$
\left\|u^{t}-u^{*}\right\|_{V} \leqslant\left\|u^{\lfloor t\rfloor}-u^{*}\right\|_{V} \rightarrow 0 \quad \text { as } t \rightarrow \infty .
$$

The continuity of $(u, t) \mapsto u^{t}$ in $X$ comes from the continuity of $(u, H) \mapsto u^{H}$ and of $t \mapsto$ $H_{t}$.

\section{$3 \quad$ Symmetry and variational principles}

Symmetrization allows to restrict the search of a minimizer to the subset of symmetric functions. Similarly we show here that on certain critical levels, there is a critical point which is symmetric. Let us first recall a general minimax principle.

Theorem 3.1 (Willem [15]). Let $X$ be a Banach space. Let $M_{0}$ be a closed subspace of the metric space $M$ and $\Gamma_{0} \subset C\left(M_{0}, X\right)$. Define

$$
\Gamma=\left\{\gamma \in C(M, X)|\gamma|_{M_{0}} \in \Gamma_{0}\right\} .
$$

If $\varphi \in C^{1}(X, \mathbb{R})$ satisfies

$$
\infty>c=\inf _{\gamma \in \Gamma} \sup _{t \in M} \varphi(\gamma(t))>a=\sup _{\gamma_{0} \in \Gamma_{0}} \sup _{t \in M_{0}} \varphi\left(\gamma_{0}(t)\right)
$$

then for every $\varepsilon \in] 0,(c-a) / 2[, \delta>0$ and $\gamma \in \Gamma$ such that

$$
\sup _{M} \varphi \circ \gamma \leqslant c+\varepsilon,
$$

there exists $u \in X$ such that

a) $c-2 \varepsilon \leqslant \varphi(u) \leqslant c+2 \varepsilon$,

b) $\operatorname{dist}(u, \gamma(M)) \leqslant 2 \delta$,

c) $\left\|\varphi^{\prime}(u)\right\| \leqslant 8 \varepsilon / \delta$. 
Remark 3.2. A slight modification of the proof gives the better estimate

$$
\operatorname{dist}\left(u, \gamma(M) \cap \varphi^{-1}([c-2 \varepsilon, c+2 \varepsilon]) \leqslant 2 \delta .\right.
$$

Theorem 3.1 yields a Palais-Smale sequence $\left(u_{n}\right)_{n \geqslant 1}$ such that $\varphi^{\prime}\left(u_{n}\right) \rightarrow 0$ and $\varphi\left(u_{n}\right) \rightarrow c$. This is an important step in order to prove that $c$ is a critical value of $\varphi$. This is the case if $\varphi$ satisfies the $(P S)_{c}$ condition: any sequence $\left(u_{n}\right)$ such that $\varphi^{\prime}\left(u_{n}\right) \rightarrow 0$ and $\varphi\left(u_{n}\right) \rightarrow c$ contains a subsequence that converges strongly.

It should be possible to have more information on the symmetry of $u$ under Assumption 2.25 provided $\varphi\left(u^{*}\right) \leqslant \varphi(u)$. A naive idea consists in replacing the path $\gamma$ by is its symmetrization $\gamma^{*}: t \in M \mapsto \gamma(t)^{*}$. Then $u$ given by Theorem 3.1 would be near of the set $\gamma^{*}(M)$. Unfortunately, when $N>1, X=W^{1, p}\left(\mathbb{R}^{N}\right)$ and $*$ is the Schwarz symmetrization, * is not continuous on $X[1]$ so that the symmetrized path $\gamma^{*}$ could be discontinuous.

This idea works if the symmetrization is approximated uniformly by continuous transformations. The convergence of the approximation scheme of the symmetrization $*$ by polarizations of Theorem 2.32 is not uniform; it becomes uniform by an appropriate change of variable.

Proposition 3.3. Suppose $M$ is a metric space, $M_{0}$ and $M_{1}$ are disjoint closed sets of $M$ and $\gamma \in C(M, X)$. Suppose that $X, V, *$ and $\mathcal{H}_{*}$ satisfy Assumption 2.25, $H_{0} \in \mathcal{H}_{*}$ and $\gamma(M) \subset S$. For any $\varepsilon>0$, there exists $\tilde{\gamma} \in C(M, X)$ such that

$$
\begin{array}{ll}
\tilde{\gamma}(t)=\gamma(t)^{H_{1} \ldots H_{\lfloor\theta\rfloor} H_{\theta}} & \forall t \in M, \text { with } \theta \geqslant 0 \text { and } H_{\tau} \in \mathcal{H}_{*} \text { for } \tau \geqslant 0, \\
\tilde{\gamma}(t)=\gamma(t)^{H_{0}} & \forall t \in M_{0}, \\
\left\|\tilde{\gamma}(t)-\gamma(t)^{*}\right\|_{V} \leqslant \varepsilon & \forall t \in M_{1} .
\end{array}
$$

Proof. For any $t \in M_{1}$, let $\delta_{t}$ be such that $B\left(t, \delta_{t}\right) \cap M_{0}=\phi$ and such that for all $s \in B\left(t, \delta_{t}\right)$, $\|\gamma(s)-\gamma(t)\|_{V} \leqslant \varepsilon / 3$ (this is possible because $\gamma$ is continuous and $X$ is continuously embedded in $V$ ). For every $t \in M_{1}$, there exists $\theta_{t}$ such that $\left\|\gamma(t)^{\theta}-\gamma(t)^{*}\right\|_{V} \leqslant \varepsilon / 3$ for $\theta \geqslant \theta_{t}$, with the notation of Proposition 2.32. The collection $\mathcal{O}=\left\{M \backslash M_{1}\right\} \cup\left\{B\left(t, \delta_{t}\right)\right\}_{t \in M_{1}}$ forms an open covering of the metric space $M$. There exists thus a partition of the unity $\left(\rho_{i}\right)_{i \in \mathcal{O}}$ subordinate to this covering [10, Theorems (T2, XXII, 5; 1) and (T2, XXII, 5; 5)]. Let $\Theta(t)=\sum_{s \in M_{1}} \rho_{s}(t) \theta_{s}$. The function $\Theta$ is continuous. Let $\tilde{\gamma}(t)=\gamma(t)^{\Theta(t)}$. If $t \in M_{0}$, then $\Theta(t)=0$ and $\tilde{\gamma}(t)=\gamma(t)^{H_{0}}$. If $t \in M_{1}$, there exists $s \in M$ such that $\rho_{s}(t)>0$ and $\theta_{s} \leqslant \Theta(t)$; hence by Proposition 2.31

$$
\begin{aligned}
\left\|\tilde{\gamma}(t)-\gamma(t)^{*}\right\|_{V} \leqslant\|\tilde{\gamma}(t)-\tilde{\gamma}(s)\|_{V}+\left\|\tilde{\gamma}(s)-\gamma(s)^{*}\right\|_{V}+\left\|\gamma(s)^{*}-\gamma(t)^{*}\right\|_{V} \\
\leqslant\left\|\gamma(s)^{\Theta(t)}-\gamma(s)^{*}\right\|_{V}+2\|\gamma(s)-\gamma(t)\|_{V} \leqslant \varepsilon
\end{aligned}
$$

since $t \in B\left(s, \delta_{s}\right)$ implies $\|\gamma(s)-\gamma(t)\|_{V} \leqslant \varepsilon / 3$.

Corollary 3.4 (Uniform approximation of symmetrization). For any $\varepsilon>0$, there exists a continuous mapping $T: S \rightarrow S$ such that $\left\|T u-u^{*}\right\|_{V}<\varepsilon$ for each $u \in S$.

Proof. Apply Proposition 3.3 with $M_{0}=\phi, M=M_{1}=S$ and $\gamma(u)=u$. Let $T u=\tilde{\gamma}(u)$.

We can now state and prove a symmetric variational principle. 
Theorem 3.5 (Symmetric variational principle). Suppose $X, V$, * and $\mathcal{H}_{*}$ satisfy Assumption 2.25. Let $M_{0}$ be a closed subspace of the metric space $M$ and $\Gamma_{0} \subset C\left(M_{0}, X\right)$. Define

$$
\Gamma=\left\{\gamma \in C(M, X)|\gamma|_{M_{0}} \in \Gamma_{0}\right\}
$$

If $\varphi \in C^{1}(X, \mathbb{R})$ satisfies

$$
\infty>c=\inf _{\gamma \in \Gamma} \sup _{t \in M} \varphi(\gamma(t))>a=\sup _{\gamma_{0} \in \Gamma_{0}} \sup _{t \in M_{0}} \varphi\left(\gamma_{0}(t)\right)
$$

and if for any $H \in \mathcal{H}_{*}$ and $u \in S, \varphi\left(u^{H}\right) \leqslant \varphi(u)$, then for every $\left.\varepsilon \in\right] 0,(c-a) / 2[, \delta>0$ and $\gamma \in \Gamma$ such that

(i) $\sup _{M} \varphi \circ \gamma \leqslant c+\varepsilon$,

(ii) $\gamma(M) \subset S$,

(iii) there exists $H_{0} \in \mathcal{H}_{*}$ such that $\left.\gamma\right|_{M_{0}}{ }^{H_{0}} \in \Gamma_{0}$,

there exists $u \in X$ such that

a) $c-2 \varepsilon \leqslant \varphi(u) \leqslant c+2 \varepsilon$,

b) $\left\|u-u^{*}\right\|_{V} \leqslant 2(2 K+1) \delta$,

c) $\left\|\varphi^{\prime}(u)\right\|_{X^{\prime}} \leqslant 8 \varepsilon / \delta$,

where $K$ is the norm of the injection of $X$ into $V$.

Proof. Without loss of generality, we can assume that $c-2 \varepsilon>a$. Let $M_{1}=(\varphi \circ \gamma)^{-1}([c-$ $2 \varepsilon, c+\varepsilon])$. This set is clearly closed. Proposition 3.3, yields a path $\tilde{\gamma} \in C(M, X)$ such that (3.1) holds with $\delta$ in place of $\varepsilon$. Theorem 3.1 with $\tilde{\gamma}$ in place of $\gamma$ gives $u$ such that

a) $c-2 \varepsilon \leqslant \varphi(u) \leqslant c+2 \varepsilon$,

b) $\operatorname{dist}\left(u, \tilde{\gamma}\left(M_{1}\right)\right) \leqslant \operatorname{dist}\left(u, \tilde{\gamma}(M) \cap \varphi^{-1}([c-2 \varepsilon, c+2 \varepsilon])\right) \leqslant 2 \delta$,

c) $\left\|\varphi^{\prime}(u)\right\| \leqslant 8 \varepsilon / \delta$.

Since the symmetrization $*$ is a contraction in $V$ by Proposition 2.31,

$$
\left\|u-u^{*}\right\|_{V} \leqslant \inf _{t \in M_{1}}\left(\|u-\tilde{\gamma}(t)\|_{V}+\left\|\tilde{\gamma}(t)-\gamma(t)^{*}\right\|_{V}+\left\|\gamma(t)^{*}-u^{*}\right\|_{V}\right) \leqslant 2(2 K+1) \delta .
$$

Informally Theorem 3.5 says that when a functional does not increase by any polarization and if the minimax construction is invariant by one polarization (existence of $H_{0}$ that preserves $\left.\Gamma_{0}\right)$, then there exists an almost symmetric Palais-Smale sequence.

It is not equivalent for a functional to decrease by symmetrization and to decrease by polarizations. In fact, many symmetrization inequalities can be proved by polarization inequalities [4]; but some inequalities, e.g. the Riesz-Sobolev inequality, hold for the symmetrization, but they do not hold for polarizations [12].

The condition $\left.\gamma\right|_{M_{0}} ^{H_{0}} \in \Gamma_{0}$ on the paths may seem weak, since it does not require invariance by symmetrization. In applications, finding such a polarizer can be impossible because of the highly noninjective character of the polarization. This imposes some kind of minimality to the energy levels on which it is possible to ensure the existence of symmetric critical points. 


\section{Applications}

\subsection{Symmetric critical points}

We first investigate the symmetry properties of solutions of the semilinear elliptic problem

$$
\begin{cases}-\Delta u+a(x) u=f(x, u) & \text { in } \Omega, \\ u=0 & \text { on } \partial \Omega,\end{cases}
$$

where $\Omega$ is a ball or an annulus and $f(x, u)=\tilde{f}(|x|, u)$ and $a(x)=\tilde{a}(|x|)$ are continuous. Those are critical points of the functional

$$
\varphi(u)=\int_{\Omega} \frac{|\nabla u|^{2}}{2}+\frac{a(x) u^{2}}{2}-F(x, u) d x
$$

defined on $H_{0}^{1}(\Omega)$, where $F(x, t)=\int_{0}^{t} f(x, s) d s$ if $t \geqslant 0$ and $F(x, t)=0$ if $t \leqslant 0$.

Here we assume

$\left(a_{1}\right) a \in L^{N / 2}(\Omega)$ if $N \geqslant 3, a \in L^{q}(\Omega)$ for $q>1$ if $N=2$ and $a \in L^{1}(\Omega)$ if $N=1$.

Under assumption $\left(a_{1}\right)$, the operator $u \mapsto-\Delta u+a(x) u$ has a nondecreasing sequence of eigenvalues $\lambda_{1} \leqslant \lambda_{2} \leqslant \ldots \leqslant \lambda_{i} \leqslant \ldots$, repeated according to their multiplicity and with associated orthonormal eigenfunctions $\left(e_{i}\right)_{i \geqslant 1}$ in $L^{2}(\Omega)[15]$.

We also assume

$\left(f_{1}\right) f \in C(\Omega \times \mathbb{R})$ and for some $1<p<2^{*}=2 N /(N-2)$ and $C>0$,

$$
|f(x, u)| \leqslant C\left(1+|u|^{p-1}\right),
$$

$\left(f_{2}\right)$ there exists $\alpha>2$ and $R>0$ such that

$$
|u| \geqslant R \Rightarrow 0<\alpha F(x, u) \leqslant u f(x, u)
$$

$\left(f_{3}\right)|f(x, u)|=o(|u|),|u| \rightarrow 0$, uniformly on $\Omega$.

Under assumption $\left(f_{1}\right)$, the functional $\varphi$ is of class $C^{1}\left(H_{0}^{1}(\Omega), \mathbb{R}\right)$. Under assumptions $\left(f_{1}\right)$ and $\left(f_{2}\right)$, the functional $\varphi$ satisfies the Palais-Smale condition: Any sequence $\left(u_{n}\right)_{n \in \mathbb{N}} \subset$ $H_{0}^{1}(\Omega)$ such that $d=\sup _{n} \varphi\left(u_{n}\right)<\infty$ and $\varphi^{\prime}\left(u_{n}\right) \rightarrow 0$ contains a convergent subsequence [15].

Consider the class

$$
\Gamma=\left\{\gamma \in C\left([0,1], H_{0}^{1}(\Omega) \mid \gamma(0)=0 \text { and } \varphi(\gamma(1))<0\right\},\right.
$$

and let

$$
c=\inf _{\gamma \in \Gamma} \sup _{t \in[0,1]} \varphi(\gamma(t)) .
$$

By the mountain pass Theorem, there is a critical point such that $\varphi(u)=c$ [15]. Under symmetry assumptions, we obtain slightly more symmetry.

Theorem 4.1. Under assumptions $\left(a_{1}\right)$ and $\left(f_{123}\right)$, if $\lambda_{1}>0, \Omega$ is a ball, $a(x) \leqslant a(y)$ and $-f(x,-s)=f(x, s) \geqslant f(y, s)$ for $x, y \in \Omega$ with $|x|_{2} \leqslant|y|_{2}$ and $s \in \mathbb{R}^{+}$, then there exists a nonnegative critical point $u$ invariant by Schwarz symmetrization such that $\varphi(u)=c$. 
Proof. For each $n \geqslant 1$, let $\gamma \in \Gamma$ be such that

$$
\max _{t \in[0,1]} \varphi(\gamma(t)) \leqslant c+1 / n
$$

Since $\varphi\left(u_{+}\right) \leqslant \varphi(u)$, we can assume $\gamma(t) \geqslant 0$ for each $t \in[0,1]$. Theorem 3.5 with $\delta=1 / n^{1 / 2}$ and $\varepsilon=1 / n$ yields $u_{n} \in H_{0}^{1}(\Omega)$ such that $\left|\varphi\left(u_{n}\right)-c\right| \leqslant 2 / n,\left\|\varphi^{\prime}\left(u_{n}\right)\right\|_{H_{0}^{-1}(\Omega)} \leqslant 8 / n^{1 / 2}$ and $\left\|u_{n}-u_{n}^{*}\right\|_{L^{2}(\Omega)} \leqslant 2(2 K+1) / n^{1 / 2}$, where $*$ denotes the Schwarz symmetrization. Since $\varphi$ satisfies the Palais-Smale condition, up to a subsequence, $u_{n} \rightarrow u$ in $H_{0}^{1}(\Omega)$, with $\varphi(u)=c$, $\varphi^{\prime}(u)=0$ and $u=u^{*}$.

Remark 4.2. The method of proof is robust with respect to changes in the minimax principle. If $\Gamma$ was defined as

$$
\Gamma=\{\gamma \in C([0,1] \mid \gamma(0)=0 \text { and } \gamma(1)=e\},
$$

where $e \in H_{0}^{1}(\Omega)$ is a fixed nonnegative function and $\varphi(e)<0$, then the conclusions of Theorem 4.1 would remain valid.

If $a$ and $f$ are slightly more regular, the moving plane method proves that any nonnegative critical point is invariant by Schwarz symmetrization. Theorem 4.1 sheds some light on the limit case where $a$ and $f$ are merely continuous functions.

If $\Omega$ is not a ball, $a$ and $f$ are not both monotone, or $f(x, \cdot)$ is not even anymore, then the moving plane method fails, but there is still some symmetry in the solutions.

Theorem 4.3. Under assumptions $\left(a_{1}\right)$ and $\left(f_{123}\right)$, if $\lambda_{1}>0, \Omega$ is a ball or an annulus, $a(x)=a(y)$ and $f(x, s)=f(y, s)$ if $x, y \in \Omega$ with $|x|_{2}=|y|_{2}$ and $s \in \mathbb{R}$, then there exists $a$ nonnegative critical point $u$ invariant by spherical cap symmetrization such that $\varphi(u)=c$.

Proof. The proof is similar to the proof of Theorem 4.1.

Remark 4.4. The method of proof is robust with respect to changes in the minimax principle. Assume $e \in H_{0}^{1}(\Omega)$ is a fixed function, $\varphi(e)<0$ and that there exists a polarizer $H_{0}$ with $0 \in \partial H_{0}$ and $u^{H_{0}}=u$. If $\Gamma$ is defined as

$$
\Gamma=\{\gamma \in C([0,1] \mid \gamma(0)=0 \text { and } \gamma(1)=e\},
$$

then the conclusions of Theorem 4.3 remain valid.

Theorem 4.3 generalizes the symmetry result of Smets and Willem for homogeneous problems [11].

If $\lambda_{1} \leqslant 0$, it is not possible anymore to obtain solutions by the mountain pass Theorem. Let $k$ be such that $\lambda_{k} \leqslant 0<\lambda_{k+1}$. Solutions of (4.1) can be obtained by Rabinowitz's linking Theorem.

Theorem 4.5 (Rabinowitz). Let $X=Y \oplus Z$ be a Banach space with $\operatorname{dim} Y<\infty$. Let $\rho>r>0$ and let $z \in Z$ be such that $\|z\|=r$. Define

$$
\begin{aligned}
M & =\{u=y+\lambda z \mid\|u\| \leqslant \rho, \lambda \geqslant 0, y \in Y\} \\
M_{0} & =\{u=y+\lambda z \mid y \in Y,\|u\|=\rho \text { and } \lambda \geqslant 0 \text { or }\|u\| \leqslant \rho \text { and } \lambda=0\}, \\
N & =\{u \in Z \mid\|u\|=r\}
\end{aligned}
$$


Let $\varphi \in C^{1}(X, \mathbb{R})$ be such that

$$
b=\inf _{N} \varphi>a=\max _{M_{0}} \varphi .
$$

If $\varphi$ satisfies the $(P S)_{c}$ condition with

$$
\begin{aligned}
c & =\inf _{\gamma \in \Gamma} \max _{u \in M} \varphi(\gamma(u)) \\
\Gamma & =\left\{\gamma \in C(M, X)|\gamma|_{M_{0}}=\mathrm{id}\right\},
\end{aligned}
$$

then $c$ is a critical value of $\varphi$.

It is a particular case of the general minimax Theorem 3.1 [15]. In order to find solutions of (4.1) assume

$\left(f_{4}\right) \lambda_{k} \frac{u^{2}}{2} \leqslant F(x, u)$ for $u \in \mathbb{R}$.

Let

$$
\begin{aligned}
& Y=\operatorname{span}\left(e_{1}, e_{2}, \ldots, e_{k}\right), \\
& Z=\left\{u \in X \mid \forall v \in Y, \int_{\Omega} u v=0\right\}, \\
& z=e_{k+1} .
\end{aligned}
$$

For some $0<r<\rho, c$ defined by (4.2) is a critical value under assumptions $\left(a_{1}\right)$ and $\left(f_{1234}\right)[15]$.

Theorem 4.6. Under assumptions $\left(a_{1}\right)$ and $\left(f_{1234}\right)$, suppose that $\Omega$ is a ball or an annulus, $a$ is Hölder-continuous and that $a(x)=a(y)$ and $f(x, s)=f(y, s)$, for each $x, y \in \Omega$ with $|x|_{2}=|y|_{2}$ and $s \in \mathbb{R}$. If $e_{1}, \ldots, e_{k}$ are radial functions, then there exists a nonnegative critical point $u$ invariant by spherical cap symmetrization $u$ such that $\varphi(u)=c$.

Proof. For each $n \geqslant 1$, let $\gamma \in \Gamma$ be such that

$$
\max _{t \in M} \varphi(\gamma(t)) \leqslant c+1 / n
$$

Since $e_{1}, \ldots, e_{k}$ are radial and since by Lemma $4.7 e_{k+1}$ is invariant with respect to a spherical cap symmetrization, there exists $H_{0}$ such that for any $u \in\left(Y+\mathbb{R}^{+} e_{k+1}\right), u^{H_{0}}=u$. Hence if $\gamma_{0} \in \Gamma_{0}$, then $\gamma_{0}^{H_{0}} \in \Gamma_{0}$. Theorem 3.5 with $\delta=1 / n^{1 / 2}$ and $\varepsilon=1 / n$ yields $u_{n} \in H_{0}^{1}(\Omega)$ such that $\left|\varphi\left(u_{n}\right)-c\right| \leqslant 2 / n,\left\|\varphi^{\prime}\left(u_{n}\right)\right\|_{H_{0}^{-1}(\Omega)} \leqslant 8 / n^{1 / 2}$ and $\left\|u_{n}-u_{n}^{*}\right\|_{L^{2}(\Omega)} \leqslant K / n^{1 / 2}$, where * denotes a spherical cap symmetrization. Since $\varphi$ satisfies the Palais-Smale condition, up to a subsequence $u_{n} \rightarrow u$ in $H_{0}^{1}(\Omega)$, with $\varphi(u)=c, \varphi^{\prime}(u)=0$ and $u=u^{*}$.

Lemma 4.7. If a is Hölder-continuous and radial, and $e_{i}$ is radial for each $1 \leqslant i \leqslant k$, there exists $P \in S^{N-1}$ such that $e_{k+1}$ is invariant under the spherical cap symmetrization with respect to $P$.

Proof. The proof is a slight variation on a proof of Bartsch, Weth and Willem [2]. Recall that $e_{k+1}$ minimizes

$$
R(u)=\int_{\Omega}|\nabla u|^{2}+a(x)
$$


on the set

$$
V=\left\{u \in H_{0}^{1}(\Omega) \mid\|u\|_{L^{2}(\Omega)}=1 \text { and } \int_{\Omega} u e_{i}=0 \text { for } 1 \leqslant i \leqslant k\right\} .
$$

Any minimizer $u$ of $R$ on $V$ satisfies the equation

$$
-\Delta u+a(x) u=\lambda_{k+1} u,
$$

with $R(u)=\lambda_{k+1}$. Since $a$ is Hölder continuous, by standard regularity estimates, $u$ is twice differentiable and is continuous up to the boundary.

Let $H$ be a polarizer such that $0 \in \partial H$. One checks that $e_{k+1}^{H} \in V$ since the eigenfunctions $e_{i}$ are radial for $1 \leqslant i \leqslant k$ and that $R\left(e_{k+1}^{H}\right)=R\left(e_{k+1}\right)$. Therefore $e_{k+1}^{H}$ is a minimizer of $R$ on $V$, and it satisfies the equation (4.4). The function $e_{k+1}$ and $e_{k+1}^{H}$ are thus both twice continuously differentiable and continuous up to the boundary. For $x \in H \cap \Omega, \mid e_{k+1}(x)-$ $e_{k+1}\left(x_{H}\right) \mid=2 e_{k+1}{ }^{H}-\left(e_{k+1}(x)+e_{k+1}\left(x_{H}\right)\right)$. Therefore $\left|e_{k+1}-e_{k+1}{ }^{H}\right| \in C^{2}(H \cap \Omega) \cap$ $C_{0}(\overline{H \cap \Omega})$. Since $e_{k+1}(\cdot H)$ also solves (4.4),

$$
\begin{aligned}
-\Delta\left|e_{k+1}(x)-e_{k+1}\left(x_{H}\right)\right|+\left(a_{H}(x)-\lambda_{k+1}\right)_{+} & \left|e_{k+1}(x)-e_{k+1}\left(x_{H}\right)\right| \\
& =\left(a(x)-\lambda_{k+1}\right)_{-}\left|e_{k+1}(x)-e_{k+1}\left(x_{H}\right)\right| \geqslant 0 .
\end{aligned}
$$

By the strong maximum principle either $\left|u-u_{H}\right|=0$ on $\Omega$, or $\left|u-u_{H}\right|>0$ on the interior of $H \cap \Omega$.

Now take $x_{0}$ in the interior of $\Omega$ such that

$$
e_{k+1}\left(x_{0}\right)=\max \left\{u(x)|x \in \Omega,| x|=| x_{0} \mid\right\} .
$$

For any polarizer such that $x_{0}$ is in the interior of $H$ and $0 \in \partial H$, by the preceding reasoning, $u^{H}=u$. Hence $u$ is invariant by spherical cap symmetrization with respect to $P=x_{0} /\left|x_{0}\right|$.

It is not possible to go further in the analysis of symmetry breaking. In fact, if $\Omega$ is a ball and $e_{i}$ is not radial for some $1 \leqslant i \leqslant k$ but is spherical cap symmetric, then Theorem 3.5 is not applicable anymore since $e_{i}$ and all its rotations can not be invariant under the same spherical cap symmetrization. This obstruction remains even when the $(N-1)$-spherical cap symmetrization is replaced by any $k$-spherical cap symmetrization. This is not surprising when compared with the situation of spherical harmonics: the first eigenfunction of the Laplace-Beltrami operator on the sphere is the constant function. Then the eigenfunctions associated to the second eigenvalue are restrictions of linear functions, and depend on only one variable. For the third eigenvalue, the eigenfunctions are restrictions of harmonic polynomials of degree two: among these some depend up to rotation on only one variable (the zonal harmonics), but some others depend on all the variables (since spherical harmonics of degree $n$ are restrictions of homogeneous harmonic polynomials of degree $n$, this follows from Proposition 4.8). This explains why it is not possible to prove any symmetry properties of eigenfunctions of $-\Delta+a(x)$ for eigenvalues above the first nonradial eigenfunction. This suggests that when $e_{i}$, for some $1 \leqslant i \leqslant k$ is not radial, a critical point of a nonlinear problem could be noninvariant with respect to any nontrivial rotation group.

Proposition 4.8. There exists a homogeneous harmonic polynomial h of degree two such that the group $G$ of linear isometries $T$ of $\mathbb{R}^{N}$ that satisfy $h \circ T=h$ is generated by the reflections with respect to $N$ orthogonal hyperplanes. In particular, $G$ is finite. 
Proof. In general if a function $f \in C^{1}\left(\mathbb{R}^{N}\right)$ is invariant with respect to a linear isometry $T$ if and only if for any $x \in \mathbb{R}^{N}, \nabla f=T^{*} \nabla f(T x)$, where $T^{*}$ denotes the adjoint of $T$. If $h$ is a second order harmonic polynomial, it can be written as $h(x)=x \cdot A x$, where $A: \mathbb{R}^{N} \rightarrow \mathbb{R}^{N}$ is linear and selfadjoint. The polynomial $h$ is invariant with respect to $T$ if and only if for each $x \in \mathbb{R}^{N}, 2 A x=2 T^{*} A T x$, i.e. $T A=A T$. Choose $A$ with eigenvalues of multiplicity one and vanishing trace. Since $A$ and $T$ commute, the eigenvectors of $A$ must be eigenvectors of $T$. Since $T$ is an isometry, $T v=v$ or $T v=-v$ for each eigenvector $v$ of $A$. Therefore if $h$ is invariant with respect to $T$, then $T$ is in the group generated by reflections with respect to hyperplanes orthogonal to the eigenvectors of $A$.

The method of this section is also adapted to Neumann boundary conditions. If the functional $\varphi$ is defined on the set $H^{1}(\Omega)$ in place of $H_{0}^{1}(\Omega)$, then the critical points of $\varphi$ are weak solutions of

$$
\begin{cases}-\Delta u+a(x) u=f(x, u) & \text { in } \Omega, \\ \partial u / \partial n=0 & \text { on } \partial \Omega .\end{cases}
$$

We are in the setting of Example 2.29; Theorems 4.3 and 4.6 remain valid for the new functional $\varphi$.

\subsection{Noncompact problems}

Consider the following semilinear partial differential equation:

$$
\left\{\begin{array}{l}
-\Delta u+V(x) u=f(x, u) \quad \text { in } \mathbb{R}^{N}, \\
u \geqslant 0,
\end{array}\right.
$$

where $f \in C\left(\mathbb{R}^{N} \times \mathbb{R}^{+}\right)$. Solutions are critical points of

$$
\varphi: H^{1}\left(\mathbb{R}^{N}\right) \rightarrow \mathbb{R}: u \mapsto \frac{1}{2} \int_{\mathbb{R}^{N}}|\nabla u|^{2}+V(x)|u|^{2} d x-\int_{\mathbb{R}^{N}} F(x, u) d x,
$$

where $F(x, t)=\int_{0}^{t} f(x, s) d s$ if $t \geqslant 0$ and $F(x, t)=0$ if $t \leqslant 0$.

Such problems were treated by Rabinowitz [8] without symmetry assumptions. When the problem is invariant by rotations, solutions may be found in the space of radial functions by Palais's symmetric criticality principle [15]. But then global minimizing properties are lost. In our approach, we consider the minimax principle for the unrestricted functional

$$
c=\inf _{\gamma \in \Gamma} \max _{t \in[0,1]} \varphi(\gamma(t)),
$$

where $\Gamma=\left\{\gamma \in C\left([0,1], H^{1}\left(\mathbb{R}^{N}\right)\right) \mid \gamma(0)=0\right.$ and $\left.\varphi(\gamma(1))<0\right\}$. Therefrom, we construct an almost symmetric Palais-Smale sequence. This proves that $c$ is a symmetric critical level, i.e. there exists a symmetric $u \in H^{1}\left(\mathbb{R}^{N}\right)$ such that $\varphi^{\prime}(u)=0$ and $\varphi(u)=c$. This provides an alternative in some cases to concentration-compactness.

Our assumptions are:

$\left(f_{1}\right)$ There exist $C \geqslant 0,2<p<2^{*}=2 N /(N-2)$, such that for all $s \in \mathbb{R}^{+}$and $x \in \mathbb{R}^{N}$

$$
f(x, s) \leqslant C\left(|s|+|s|^{p-1}\right)
$$


$\left(f_{2}\right)$ there exists $x \in \mathbb{R}^{N}$ and $s>0$ such that $F(x, s)>0$,

$\left(f_{3}\right)$ there exists $\alpha>2$ such that for each $x \in \mathbb{R}^{N}$ and $s \in \mathbb{R}^{+}$,

$$
\alpha F(x, s) \leqslant s f(x, s)
$$

$\left(f_{4}\right)$ for $x, y \in \mathbb{R}^{N}$, if $|x| \leqslant|y|$ then for all $s \in \mathbb{R}^{+}, f(x, s) \geqslant f(y, s)$,

$\left(f_{5}\right) f(x, s)=o(|s|)$, as $|s| \rightarrow 0$, uniformly in $x \in \mathbb{R}^{N}$,

$\left(V_{1}\right)$ there exists $m, M \in \mathbb{R}$ such that for any $x \in \mathbb{R}^{N}, 0<m \leqslant V(x) \leqslant M$,

$\left(V_{2}\right)$ for $x, y \in \mathbb{R}^{N}$, if $|x| \leqslant|y|$ then $V(x) \leqslant V(y)$.

Remark 4.9. The condition $V(x) \leqslant M$ can be dropped provided that the functional $\varphi$ is defined on the subset of functions $u$ of $H_{0}^{1}(\Omega)$ such that $\int_{\Omega} V(x) u^{2} d x<\infty$.

Theorem 4.10. Under the preceding assumptions, $c$ is a critical value and there is a radial symmetric decreasing critical point $u$ such that $\varphi(u)=c$.

Lemma 4.11. Under assumptions $\left(f_{12345}\right)$ and $\left(V_{12}\right)$, there exists a sequence $\left(u_{n}\right)_{n \geqslant 1} \subset$ $H^{1}\left(\mathbb{R}^{N}\right)$ such that

$$
\begin{aligned}
\varphi\left(u_{n}\right) & \rightarrow c, & & \\
\varphi^{\prime}\left(u_{n}\right) & \rightarrow 0 & & \text { strongly in } H^{-1}\left(\mathbb{R}^{N}\right), \\
u_{n}-u_{n}^{*} & \rightarrow 0 & & \text { in }\left(L^{2} \cap L^{2^{*}}\right)(\Omega),
\end{aligned}
$$

where * denotes the Schwarz symmetrization.

Proof. Note first the set $\Gamma=\left\{\gamma \in C\left([0,1], H^{1}\left(\mathbb{R}^{N}\right)\right) \mid \gamma(0)=0\right.$ and $\left.\varphi(\gamma(1))<0\right\}$ is not empty. From assumptions $\left(f_{2}\right)$ and $\left(f_{3}\right)$, there exists $K_{1}$ and an open set $U \subset \mathbb{R}^{N}$ such that for $x \in U$ and $s \in \mathbb{R}^{+}$,

$$
F(x, s) \geqslant K_{1}\left(|s|^{\alpha}-1\right),
$$

Let $u \in \mathcal{D}_{+}(U)$ be nonzero. For any $\tau \geqslant 0$

$$
\varphi(\tau u) \leqslant \frac{\tau^{2}}{2} \int_{\mathbb{R}^{N}}|\nabla u|^{2}+V(x) u^{2} d x-\tau^{\alpha} K_{1}\|u\|_{\alpha}^{\alpha}+K_{1} \mathcal{L}^{N}(\operatorname{supp} u) .
$$

Since $\alpha>p$, there exists $\bar{\tau}>0$ such that $\varphi(\bar{\tau} u)<0$. Let $\gamma(t)=u t / \bar{\tau}$. It is clear that $\gamma \in \Gamma$.

By assumptions $\left(f_{4}\right)$ and $\left(f_{5}\right)$, there is $C^{\prime}>0$ such that $|f(x, s)| \leqslant m|s| / 2+C^{\prime}|s|^{p-1}$. That implies

$$
\begin{aligned}
\varphi(u) \geqslant \min (1, m) \frac{\|u\|_{H^{1}}^{2}}{2}-\frac{\|u\|_{L^{2}}^{2}}{2}-C^{\prime} \frac{\|u\|_{L^{p}}^{p}}{p} & \\
& \geqslant(\min (1, m)-m / 2) \frac{\|u\|_{H^{1}}^{2}}{2}-C^{\prime \prime} \frac{\|u\|_{H^{1}}^{p}}{p} .
\end{aligned}
$$

Therefore, there exists $\rho>0$ such that $\varphi(u) \geqslant 0$ if $\|u\|_{H^{1}} \leqslant \rho$ and $\varphi(u) \geqslant \mu>0$ if $\|u\|_{H^{1}}=\rho$. Hence if $\gamma \in \Gamma,\|\gamma(1)\|_{H^{1}}>\rho$ and so $\max _{t \in[0,1]} \varphi(\gamma(t)) \geqslant \mu>0$. This shows that $c>a$ in Theorem 3.5. For any polarizer, by Proposition 2.19, $\varphi\left(u^{H}\right)=\varphi(u)$. Let $H_{0}$ be any fixed polarizer. Then $\gamma(0)^{H_{0}}=0=\gamma(0)$ and $\varphi\left(\gamma(1)^{H_{0}}\right) \leqslant \varphi(\gamma(1))$. The conclusions follow from the symmetric minimax principle (Theorem 3.5). 
Proof of Theorem 4.10. Let $\left(u_{n}\right)$ be the sequence given by Lemma 4.11. For sufficiently large $n$, we have

$$
1+c+\left\|u_{n}\right\|_{H^{1}\left(\mathbb{R}^{N}\right)} \geqslant \varphi\left(u_{n}\right)-\frac{1}{\alpha}\left\langle\varphi^{\prime}\left(u_{n}\right), u_{n}\right\rangle \geqslant\left(\frac{1}{2}-\frac{1}{\alpha}\right)\left\|u_{n}\right\|_{H^{1}\left(\mathbb{R}^{N}\right)}^{2} ;
$$

since $\alpha>2$, the sequence $\left(u_{n}\right)$ is bounded in $H^{1}\left(\mathbb{R}^{N}\right)$.

The sequence $\left(u_{n}^{*}\right)$ is also bounded in $H^{1}\left(\mathbb{R}^{N}\right)$ by the Polya-Szegö inequality (see e.g. [4]) and by Strauss' Theorem [15], $\left(u_{n}^{*}\right)$ is compact in $L^{p}\left(\mathbb{R}^{N}\right)$. Finally, since $\left\|u_{n}-u_{n}^{*}\right\|_{p} \rightarrow 0$, the sequence $\left(u_{n}\right)$ is also compact in $L^{p}\left(\mathbb{R}^{N}\right)$. We can thus suppose that $u_{n} \rightarrow u$ weakly in $H^{1}\left(\mathbb{R}^{N}\right)$ and strongly in $L^{p}\left(\mathbb{R}^{N}\right)$.

Finally, we need to prove that

$$
\int_{\mathbb{R}^{N}}\left(f\left(x, u_{n}\right)-f(x, u)\right)\left(u_{n}-u\right) d x \rightarrow 0
$$

as $n \rightarrow \infty$. By $\left(f_{4}\right)$ and $\left(f_{5}\right)$, for any $\varepsilon>0$, there is $c_{\varepsilon}$ such that

$$
|f(x, s)| \leqslant \varepsilon|s|+c_{\varepsilon}|s|^{p-1} .
$$

Then

$$
\begin{aligned}
& \limsup _{n \rightarrow \infty}\left|\int_{\mathbb{R}^{N}}\left(f\left(x, u_{n}\right)-f(x, u)\right)\left(u_{n}-u\right) d x\right| \\
& \quad \leqslant 2 \varepsilon\|u\|_{H^{1}\left(\mathbb{R}^{N}\right)}^{2}+2 c_{\varepsilon}\|u\|_{L^{p}\left(\mathbb{R}^{N}\right)}^{p-1} \limsup _{n \rightarrow \infty}\left\|u_{n}-u\right\|_{L^{p}\left(\mathbb{R}^{N}\right)},
\end{aligned}
$$

and our claim is proved since $u$ is in $H^{1}\left(\mathbb{R}^{N}\right)$ and converges in $L^{p}\left(\mathbb{R}^{N}\right)$. Since the sequence $\left(u_{n}\right)$ is Palais-Smale, by standard arguments, $u_{n} \rightarrow u$ in $H^{1}\left(\mathbb{R}^{N}\right)$ and thus $u$ is a critical point of $\varphi$ and $\varphi(u)=c$. Furthermore $u^{*}=u$.

\section{Acknowledgment}

The author thanks Michel Willem for encouragements and discussions. The author is supported by a research fellow grant of the Belgian Fonds National de la Recherche Scientifique.

\section{References}

[1] F. J. Almgren, Jr. and E. H. Lieb, The (non) continuity of symmetric decreasing rearrangement, Symposia Mathematica, Vol. XXX (Cortona, 1988), Sympos. Math., XXX, Academic Press, London, 1989, pp. 89-102.

[2] T. Bartsch, T. Weth, and M. Willem, Partial symmetry of least energy nodal solutions to some variational problems, Journal d'Analyse Mathématique, to appear.

[3] F. Brock, Continuous rearrangement and symmetry of solutions of elliptic problems, Proc. Indian Acad. Sci. Math. Sci. 110 (2000), no. 2, 157-204.

[4] F. Brock and A. Y. Solynin, An approach to symmetrization via polarization, Trans. Amer. Math. Soc. 352 (2000), no. 4, 1759-1796. 
[5] A. Burchard, Steiner symmetrization is continuous in $W^{1, p}$, Geom. Funct. Anal. 7 (1997), no. $5,823-860$.

[6] L. E. Fraenkel, An introduction to maximum principles and symmetry in elliptic problems, Cambridge University Press, Cambridge, 2000.

[7] B. Gidas, W. M. Ni, and L. Nirenberg, Symmetry and related properties via the maximum principle, Comm. Math. Phys. 68 (1979), no. 3, 209-243.

[8] P. H. Rabinowitz, On a class of nonlinear Schrödinger equations, Z. Angew. Math. Phys. 43 (1992), no. 2, 270-291.

[9] J. Sarvas, Symmetrization of condensers in n-space., Ann. Acad. Sci. Fenn., Ser. A I 522 (1972), $44 \mathrm{p}$.

[10] L. Schwartz, Analyse. Deuxième partie: Topologie générale et analyse fonctionnelle, Collection Enseignement des Sciences, no. 11, Hermann, Paris, 1970.

[11] D. Smets and M. Willem, Partial symmetry and asymptotic behavior for some elliptic problems, Calc. Var. Partial Differential Equations 18 (2003), no. 1, 57-75.

[12] J. Van Schaftingen, Anisotropic symmetrizations, in preparation.

[13] _ Universal approximation of symmetrizations by polarizations, Proc. Amer. Math. Soc., accepted.

[14] J. Van Schaftingen and M. Willem, Set transformations, symmetrizations and isoperimetric inequalities, Nonlinear Analysis and Applications to the Physical Sciences (V. Benci and A. Masiello, eds.), Springer, 2004, pp. 135-152.

[15] M. Willem, Minimax theorems, Progress in Nonlinear Differential Equations and their Applications, vol. 24, Birkhäuser, Boston, Mass., 1996. 